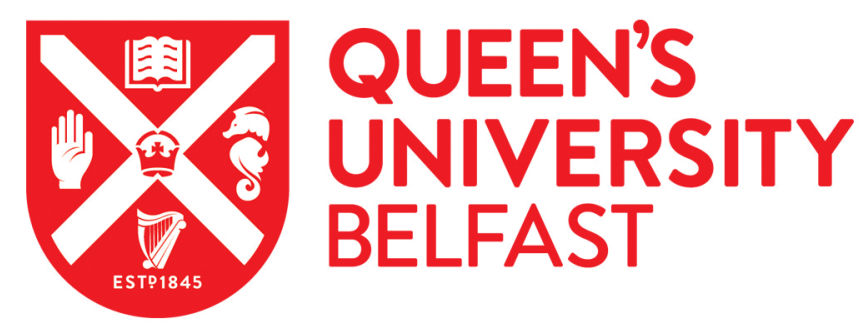

\title{
Phosphorylation of synaptic GTPase-activating protein (synGAP) by polo-like kinase (Plk2) alters the ratio of its GAP activity toward HRas, Rap1 and Rap2 GTPases.
}

Walkup IV, W. G., Sweredoski, M. J., Graham, R., Hess, S., \& Kennedy, M. B. (2018). Phosphorylation of synaptic GTPase-activating protein (synGAP) by polo-like kinase (Plk2) alters the ratio of its GAP activity toward HRas, Rap1 and Rap2 GTPases. Biochemical and Biophysical Research Communications, 503(3), 1599-1604. https://doi.org/10.1016/j.bbrc.2018.07.087

\section{Published in:}

Biochemical and Biophysical Research Communications

\section{Document Version:}

Peer reviewed version

Queen's University Belfast - Research Portal:

Link to publication record in Queen's University Belfast Research Portal

\section{Publisher rights}

Copyright 2018 Elsevier.

This manuscript is distributed under a Creative Commons Attribution-NonCommercial-NoDerivs License

(https://creativecommons.org/licenses/by-nc-nd/4.0/), which permits distribution and reproduction for non-commercial purposes, provided the author and source are cited.

\section{General rights}

Copyright for the publications made accessible via the Queen's University Belfast Research Portal is retained by the author(s) and / or other copyright owners and it is a condition of accessing these publications that users recognise and abide by the legal requirements associated with these rights.

Take down policy

The Research Portal is Queen's institutional repository that provides access to Queen's research output. Every effort has been made to ensure that content in the Research Portal does not infringe any person's rights, or applicable UK laws. If you discover content in the

Research Portal that you believe breaches copyright or violates any law, please contact openaccess@qub.ac.uk. 


\section{Highlights}

1. Phosphorylation by Plk2 regulates Ras and Rap specificity of synGAP's GAP activity.

2. Phosphorylation by both Plk2 and CDK5 additively increases RasGAP activity by $230 \%$.

3. Together, CaMKII, Plk2, and CDK5 act like a rheostat to finely tune GAP specificity.

\section{Title}

Phosphorylation of Synaptic GTPase-activating Protein (synGAP) by Polo-Like Kinase (Plk2) alters the Ratio of Its GAP Activity toward HRas, Rap1 and Rap2 GTPases

\section{Authors}

Ward G. Walkup 4th, ${ }^{\mathrm{a}, \mathrm{b},{ }^{*}}$, Michael J. Sweredoski ${ }^{\mathrm{c}}$, Robert L. Graham ${ }^{\mathrm{c}, \mathrm{d}}$, Sonja Hess ${ }^{\mathrm{c}, \mathrm{e}}$, and Mary B.

Kennedy $^{\mathrm{a}}$

${ }^{a}$ Division of Biology and Biological Engineering, California Institute of Technology, Pasadena, CA 91125

${ }^{\mathrm{b}}$ Current affiliation: De Novo Discovery, FogPharma, Cambridge, MA

${ }^{\mathrm{c}}$ Proteome Exploration Laboratory of the Beckman Institute, California Institute of Technology,

Pasadena, CA 91125

${ }^{\mathrm{d}}$ Current affiliation: Institute of Cancer Sciences, Faculty of Medical and Human Sciences, University of Manchester, UK M13 9PL

${ }^{\mathrm{e}}$ Current affiliation: Antibody Discovery and Protein Engineering, MedImmune, Gaithersburg, MD

*Corresponding author. Division of Biology and Biological Engineering 216-76, California Institute of Technology, Pasadena, CA, USA 91125 
Email address: wwalkup@alumni.caltech.edu (W.G. Walkup 4th)

\begin{abstract}
SynGAP is a Ras and Rap GTPase-activating protein (GAP) found in high concentration in the postsynaptic density (PSD) fraction from mammalian forebrain where it binds to PDZ domains of PSD95. Phosphorylation of pure recombinant synGAP by $\mathrm{Ca}^{2+} /$ calmodulin-dependent protein kinase II (CaMKII) shifts the balance of synGAP's GAP activity toward inactivation of Rap1; whereas phosphorylation by cyclin-dependent kinase 5 (CDK5) has the opposite effect, shifting the balance toward inactivation of HRas. These shifts in balance contribute to regulation of the numbers of surface AMPA receptors, which rise during synaptic potentiation (CaMKII) and fall during synaptic scaling (CDK5). Polo-like kinase 2 (Plk2/SNK), like CDK5, contributes to synaptic scaling. These two kinases act in concert to reduce the number of surface AMPA receptors following elevated neuronal activity by tagging spine-asssociated Rap-specific GAP protein (SPAR) for degradation, thus raising the level of activated Rap. Here we show that Plk2 also phosphorylates and regulates synGAP. Phosphorylation of synGAP by Plk2 stimulates its GAP activity toward HRas by $65 \%$, and toward Rap1 by $16 \%$. Simultaneous phosphorylation of synGAP by Plk2 and CDK5 at distinct sites produces an additive increase in GAP activity toward HRas ( 230\%) and a smaller, non-additive increase in activity toward Rap1 ( 15\%). Dual phosphorylation also produces an increase in GAP activity toward Rap2 ( 40-50\%), an effect not produced by either kinase alone. As we previously observed for $\mathrm{CDK} 5$, addition of $\mathrm{Ca}^{2+} / \mathrm{CaM}^{2}$ causes a substrate-directed doubling of the rate and stoichiometry of phosphorylation of synGAP by Plk2, targeting residues also phosphorylated by CaMKII. In summary, phosphorylation by PIk2, like CDK5, shifts the ratio of GAP activity of synGAP to produce a greater decrease in active Ras than in active Rap, which would produce a shift toward a decrease in the number of surface AMPA receptors in neuronal dendrites.
\end{abstract}

\title{
Key Words
}


synaptic plasticity; synaptic scaling; postsynaptic density; synaptic GTPase activating protein (synGAP); cyclin-dependent kinase 5 (CDK5); polo-like kinase 2 (Plk2)

\begin{abstract}
Abbreviations
synaptic GTPase activating protein (synGAP), soluble recombinant synGAP fragment comprising residues 103-1293 (r-synGAP), calmodulin (CaM), $\mathrm{Ca}^{2+} /$ calmodulin-dependent protein kinase II (CaMKII), cyclin-dependent kinase 5 (CDK5), polo-like kinase 2 (Plk2), p21 Ras (HRas), Ras-related protein-1 or $2(\operatorname{Rap} 1 / 2)$,
\end{abstract}

\title{
1. Introduction
}

The post synaptic density (PSD) of neurons harbors several cytosolic signaling complexes that are associated with glutamate receptors at the postsynaptic membrane of excitatory synapses [1]. synGAP, a dual Ras and Rap GTPase Activating Protein (GAP), is unusually highly concentrated in the PSD of excitatory synapses [2-4]. It binds tightly to the PDZ domains of PSD-95 [2, 5] which serves to position it in close proximity to NMDA-type and AMPA-type glutamate receptors (NMDARs and AMPARs, respectively), and allows it to regulate the composition of the PSD by restricting binding of other proteins to PSD-95 [6].

SynGAP stimulates the intrinsic GTPase activity of Rap 100-fold and that of Ras, which has a higher intrinsic rate, about 3 to 7-fold [4, 7, 8]. Stimulation of Ras and Rap by various signaling pathways [9-11] modulates AMPAR trafficking in opposite directions, with active Ras increasing insertion (exocytosis) of AMPARs at the dendritic membrane, while active Rap increases their removal (endocytosis) [9].

Activation of NMDARs in cultured CNS neurons leads to phosphorylation of synGAP by CaMKII $[2,11$, 12]. We showed that a recombinant, purified, soluble synGAP- $\alpha 1$ that lacks 102 residues at the $\mathrm{N}$ 
terminus (r-synGAP) can be expressed in soluble form and phosphorylated by purified $\mathrm{Ca}^{2+} /$ calmodulindependent protein kinase II (CaMKII) and cyclin-dependent kinase 5 (CDK5) [4]. Phosphorylation by CaMKII accelerates the rate of inactivation of Rap1 more potently than the rate of inactivation of HRas, whereas phosphorylation by CDK5 has the opposite effect [4].

Within postsynaptic spines, Spine-associated RapGAP (SPAR), also regulates Rap [13]. SPAR acts differently than synGAP because it stimulates the activity of Rap2 more potently than Rap1. CDK5 and polo-like kinase 2 (Plk2/SNK) act in concert to regulate SPAR; CDK5 primes phosphorylation by Plk2, ultimately tagging SPAR for degradation $[14,15]$. During homeostatic down-regulation of synapses, loss of SPAR activity increases the steady-state level of active Rap, thus increasing endocytosis of surface AMPA receptors.

We previously showed that Plk2 phosphorylation of r-synGAP decreases its affinity for PSD-95 [6] and that CDK5 phosphorylation of r-synGAP accelerates its HRas GAP activity [4]. Here we extend those studies by examining the effect of phosphorylation by Plk2 on GAP activity of r-synGAP and the effect of simultaneous phosphorylation of r-synGAP by Plk2 and CDK5. We determine the location and stoichiometry of Plk2 phosphorylation sites in r-synGAP, and the effect of their phosphorylation on HRas and Rap1/Rap2 GAP activity. We show that, as for CDK5, the addition of $\mathrm{Ca}^{2+} / \mathrm{CaM}$ produces a substrate-directed increase in phosphorylation. Finally, we show that simultaneous phosphorylation by CDK5 and Plk2 results in a large additive increase in GAP activity toward HRas and a much smaller increase in GAP activity toward both Rap1 and Rap2.

\section{Materials and Methods}

2.1 Expression and Purification of Recombinant Proteins. R-synGAP, residues 103-1293 in synGAP A1$\alpha 1$ (118-1308 in synGAP A2- $\alpha 1)$, and full length HRas, Rap1B and Rap2A were purified as previously 
described [4]. We used synGAP isoform names and residue numbering from ref. [16] with all residue numbering corresponding to synGAP A1- $\alpha 1$.

2.2 Stoichiometry and Rate of R-synGAP or $\alpha$-casein Phosphorylation by Plk2. R-synGAP (286 nM) or dephosphorylated $\alpha$-casein $(3.7 \mu \mathrm{M})$ from bovine milk (Sigma-Aldrich) was phosphorylated with $110 \mathrm{nM}$ Plk2 (Life Technologies). Phosphorylated proteins were detected and calculation of the stoichiometry of phosphorylation was performed as described in [4].

2.3 Phosphorylation of R-synGAP by Plk2 or CDK5 for use in GTPase Assays. Phosphorylation of $725 \mathrm{nM}$ r-synGAP by $230 \mathrm{nM}$ Plk2, $230 \mathrm{nM}$ CDK5/p35 or $230 \mathrm{nM}$ Plk2 and CDK5/p35 was carried out as described in [4]. GTPase assays were carried out under conditions previously described in $[4,6]$.

2.4 Mass Spectrometry of Phosphorylated R-synGAP. Mass spectrometry of phosphorylated r-synGAP using a hybrid LTQ-FT (Thermo Scientific) equipped with a nano-electrospray ion source (Thermo Scientific) was carried out by the Proteome Exploration Laboratory at the California Institute of Technology as previously described [4].

\section{Results and Discussion}

3.1 Stoichiometry and Rate of Phosphorylation of r-SynGAP by Plk2. Both endogenous and recombinant synGAP expressed in COS7 cells [17] can be phosphorylated by Plk2. In our assay, r-synGAP was phosphorylated by Plk2 (Fig. 1A) at a rate and stoichiometry similar to that of CDK5 phosphorylation [4]. The reaction was linear for $10 \mathrm{~min}$, reaching a stoichiometry of $\sim 0.4 \mathrm{~mol}$ phosphate/mol r-synGAP. After $30 \mathrm{~min}$, the stoichiometry approached $\sim 0.8 \mathrm{~mol}$ phosphate $/ \mathrm{mol}$. As for CDK5, addition of $\mathrm{Ca}^{2+} / \mathrm{CaM}$ to Plk2 phosphorylation reactions doubles the stoichiometry and rate of r-synGAP phosphorylation to $\sim 0.8$ and $\sim 1.8$ mol phosphate/mol at 10 and 30 minutes, respectively (Fig. 1A). In contrast, phosphorylation 
by Plk2 of dephosphorylated $\alpha$-caesin, was unaffected by $\mathrm{Ca}^{2+} / \mathrm{CaM}$ (Fig. 1B). We previously showed that synGAP contains a binding site for $\mathrm{Ca}^{2+} / \mathrm{CaM}$ with affinity in the $\mathrm{nM}$ range [4]. Thus, the simplest explanation for the effect of $\mathrm{Ca}^{2+} / \mathrm{CaM}$ is that its binding to synGAP induces a conformational change that increases accessibility to CDK5 and Plk2, accelerating the kinase reaction rate and enabling the phosphorylation of additional residues.

3.2 Identification of Sites in r-SynGAP Phosphorylated by Plk2. Recombinant synGAP expressed in COS cells was phosphorylated at nine residues by Plk2 (S364, S370, S434, S451, S821, S825, S827 and S880); however, phosphorylation at only three of them (S370, S434, S825 and S827) influenced synGAP's HRas GAP activity [17]. We identified eight additional Plk2 phosphorylation sites in r-synGAP (S140, S750, S751, S756, S765, S808, S810, T897), and confirmed phosphorylation at S821, S825 and S827 in vitro (Table 1, Supplemental Table 1 and Data). All of the identified Plk2 phosphorylation sites had high Mascot scores and false localization rates of less than 1\%. Phosphorylation at sites S140, S750, S751, S756, S765, S808 was detected after 2 minutes of reaction with Plk2, whereas phosphorylation at S810, S821, S825, S827, and T897 was only detectable after 10 minutes of reaction with Plk2. We previously showed that addition of $\mathrm{Ca}^{2+} / \mathrm{CaM}$ to reactions with $\mathrm{CDK} 5 / \mathrm{p} 35$ results in phosphorylation of r-synGAP at S751, S765, S810, S1093 and S1123, in addition to sites S728, S773/T775, S802 and S842 [4] (Table 2, Supplemental Table 2 and Data). Similarly, here we show that addition of $\mathrm{Ca}^{2+} / \mathrm{CaM}$ results in phosphorylation of S810 and T897 by Plk2 within 2 min, and phosphorylation of S1099, S1123 and S1283, which are also phosphorylated by CaMKII, after 10 min $[4,12]$ (Table 1, Supplemental Table 1 and Data). Plk2 and Plk3 kinases prefer phosphorylation sites in which acidic amino acids (D, E) occur at positions between -4 and +4 of the target serine or threonine residue; in endogenous substrates, this preference is often extended to additional positions (e.g. -7 to +7 ) [18-23]. All of the Plk2 phosphorylation sites in r-synGAP, except S1123, contain one (S140, S756, S765, S808, S821, S825, S827, T897, 
S1099, S1283), two (S750, S751) or three (S810) acidic residues within -6 to +6 residues of the target serine or threonine residue.

3.3 Effect of Plk2 Phosphorylation on GAP Activity of R-synGAP. Plk2 phosphorylation increases the RasGAP activity of synGAP in COS cell lysates [17]. We examined the effect of phosphorylation of rsynGAP on its GAP activity toward Rap1, Rap2, and Ras, using the in vitro Ras and Rap GTPase assays described in [4]. As we observed for CDK5, phosphorylation by Plk2 increased the HRas GAP activity of r-synGAP substantially (70\%) (Fig. 2A), and the Rap1 GAP activity slightly (15\%) (Fig. 2B), but did not affect GAP activity of Rap2 (Fig. 2C). The effect of Plk2 on HRas GAP activity was significant after 1 min and continued to rise throughout the time course, whereas the effect on Rap1 was nearly maximal (11\%) after 2 min. Addition of $\mathrm{Ca}^{2+} / \mathrm{CaM}$ to phosphorylation reactions did not alter HRas or Rap1 GAP activity significantly, but caused a small increase in Rap2 GAP activity (11\%) after 10 min. These data show that phosphorylation of r-synGAP by Plk2 accelerates its rate of inactivation of HRas considerably more potently than inactivation of Rap1 or Rap2, which in vivo would result in a relative increase in the steady-state level of active Rap, thus increasing endocytosis of surface AMPA receptors [9].

\subsection{Effect of Concomitant CDK5 and Plk2 Phosphorylation on GAP Activity of R-synGAP. During} synaptic scaling, Plk2 binds to and phosphorylates SPAR that has previously been phosphorylated at a specific site by CDK5. The phosphorylation targets SPAR for ubiquitination, ultimately leading to a loss of mature spines and excitatory synapses $[14,15]$. Efficient ubiquitination of SPAR requires the "priming" phosphorylation by CDK5 [15]. We found that concomitant phosphorylation of r-synGAP by Plk2 and CDK5 increases its GAP activity toward HRas substantially and synergystically (230\%, Fig. 3A). It had only a slight effect on GAP activity toward Rap1 (18\%, Fig. 3B), but produced a substantial increase in GAP activity toward Rap2A that appeared more than additive (40\%, Fig. 3C). Addition of $\mathrm{Ca}^{2+} / \mathrm{CaM}$ to these reactions had no statistically significant effects on GAP activity toward HRas (Fig. 3A) or Rap1 (Fig. 3B) and a slight effect on GAP activity toward Rap2 (Fig. 3C). These data suggest that Plk2 and 
CDK5 act together to drive the synapse toward removal of AMPARs through activation of the Rap pathway by triggering removal of SPAR, and by increasing the GAP activity of r-synGAP toward HRas. Activation of Plk2 also triggers elimination of the HRas activator RasGRF1 and stimulates the Rap activator PDZGEF1 [17].

3.5 CaMKII, CDK5, and Plk2 Produce a Continuum of Changes in the Relative Levels of Ras and Rap GAP Activity of synGAP. Ras and Rap are critical regulators of the endocytosis and exocytosis of AMPARs near the synapse [9]. The steady-state levels of activity of these two GTPases are set by the balance of their activation by exchange of GDP for GTP and their inactivation by hydrolysis of the bound GTP. Phosphorylation of synGAP by CaMKII alters the ratio of inactivation of Rap1 and HRas (Fig. 4A) [4] in a direction that would shift the steady-state balance of AMPAR trafficking toward exocytosis resulting in an increase in surface AMPA receptors. Conversely, phosphorylation of synGAP by CDK5 or Plk2 alters the ratio in the opposite direction (Fig. 4A) [4], shifting the balance of AMPAR trafficking toward endocytosis of AMPA receptors and a decrease in the number of surface AMPARs. Concomitant phosphorylation by Plk2 and CDK5 drives up the rate of inactivation of HRas by synGAP even further (Fig. 4A), which would strongly drive the endocytosis of AMPARs. CaMKII is activated rapidly when $\mathrm{Ca}^{2+}$ flows through NMDA receptors during induction of LTP [1]; whereas both Plk2 and CDK5 are induced by activity-driven protein synthesis over a longer time scale [14, 24]. CDK5 activity is further limited by the availability of its p35 and p39 subunits, which are constitutively degraded and require NGF or BDNF to stimulate synthesis [25]. The absence of rapid activation mechanisms and the slow catalytic rates of CDK5 and Plk2 and are consistent with a homeostatic role. Thus, phosphorylation of synGAP by combinations of different kinases could function like a rheostat to rapidly or gradually adjust the steadystate number of surface AMPA receptors at each synapse.

In addition to altering the ratio of GAP activity toward HRas or Rap1, phosphorylation of synGAP can also alter the ratio of GAP activity toward Rap1 or Rap2 (Fig. 4B). Phosphorylation by CaMKII 
increases the ratio of GAP activity toward Rap1 versus Rap2 by markedly increasing the Rap1 GAP activity. Concomitant phosphorylation of synGAP by Plk2 and CDK5 has the opposite effect, decreasing the ratio of GAP activity toward Rap1 versus Rap2 by increasing Rap2 GAP activity. Rap1 and Rap2 are both present in pyramidal neurons in the hippocampus and cortex, but the differences in their functions are not yet clear. LTD inducing stimuli stimulate the Rap1-dependent p38 MAPK pathway, resulting in phosphorylation of AMPARs with short cytoplasmic tails (GluR2/3) and their subsequent exocytosis [9, 26], while the Rap2-dependent JNK pathway is activated by depotentiation-inducing stimuli and causes dephosphorylation of AMPARs with long cytoplasmic tails (GluR1, GluR2L) and their removal from the synapse [27, 28]. Given the degree of crosstalk between the Rap1, Rap2 and Ras pathways , and the diverse localization of small GTPases and their activators and effectors, it is plausible that distinct sub cellular pools of small GTPases may perform discrete functions in the synapse [29].

\section{Acknowledgements}

This work was supported by grants from the Gordon and Betty Moore Foundation (Center for Integrative Study of Cell Regulation), the Hicks Foundation for Alzheimer's Research, the Allen and Lenabelle Davis Foundation, and from National Institutes of Health Grant MH095095 to MBK. WGW IV was supported by the National Science Foundation Graduate Research Fellowship under Grant No. 2006019582 and National Institutes of Health under Grant No. NIH/NRSA 5 T32 GM07616. The PEL is supported by the Gordon and Betty Moore Foundation through grant GBMF775 and the Beckman Institute.

\section{References}

[1] M.B. Kennedy, Synaptic Signaling in Learning and Memory, Cold Spring Harbor perspectives in biology, 8 (2013) a016824.

[2] H.J. Chen, M. Rojas-Soto, A. Oguni, M.B. Kennedy, A synaptic Ras-GTPase activating protein (p135 SynGAP) inhibited by CaM kinase II, Neuron, 20 (1998) 895-904. 
[3] M. Sheng, C.C. Hoogenraad, The postsynaptic architecture of excitatory synapses: a more quantitative view, Annual review of biochemistry, 76 (2007) 823-847.

[4] W.G. Walkup IV, L. Washburn, M.J. Sweredoski, H.J. Carlisle, R.L. Graham, S. Hess, M.B. Kennedy, Phosphorylation of synaptic GTPase-activating protein (synGAP) by $\mathrm{Ca} 2+/$ calmodulin-dependent protein kinase II (CaMKII) and cyclin-dependent kinase 5 (CDK5) alters the ratio of its GAP activity toward Ras and Rap GTPases, The Journal of biological chemistry, 290 (2015) 4908-4927.

[5] J.H. Kim, D. Liao, L.F. Lau, R.L. Huganir, SynGAP: a synaptic RasGAP that associates with the PSD-95/SAP90 protein family, Neuron, 20 (1998) 683-691.

[6] W.G. Walkup IV, T.L. Mastro, L.T. Schenker, J. Vielmetter, R. Hu, A. Iancu, M. Reghunathan, B.D. Bannon, M.B. Kennedy, A model for regulation by SynGAP-alpha1 of binding of synaptic proteins to PDZ-domain 'Slots' in the postsynaptic density, Elife, 5 (2016).

[7] G. Krapivinsky, I. Medina, L. Krapivinsky, S. Gapon, D.E. Clapham, SynGAP-MUPP1CaMKII synaptic complexes regulate p38 MAP kinase activity and NMDA receptor-dependent synaptic AMPA receptor potentiation, Neuron, 43 (2004) 563-574.

[8] V. Pena, M. Hothorn, A. Eberth, N. Kaschau, A. Parret, L. Gremer, F. Bonneau, M.R. Ahmadian, K. Scheffzek, The C2 domain of SynGAP is essential for stimulation of the Rap GTPase reaction, EMBO reports, 9 (2008) 350-355.

[9] J.J. Zhu, Y. Qin, M. Zhao, L. Van Aelst, R. Malinow, Ras and Rap control AMPA receptor trafficking during synaptic plasticity, Cell, 110 (2002) 443-455.

[10] M.B. Kennedy, H.C. Beale, H.J. Carlisle, L.R. Washburn, Integration of biochemical signalling in spines, Nature reviews. Neuroscience, 6 (2005) 423-434.

[11] H.J. Carlisle, P. Manzerra, E. Marcora, M.B. Kennedy, SynGAP regulates steady-state and activity-dependent phosphorylation of cofilin, The Journal of neuroscience : the official journal of the Society for Neuroscience, 28 (2008) 13673-13683.

[12] J.S. Oh, P. Manzerra, M.B. Kennedy, Regulation of the neuron-specific Ras GTPaseactivating protein, synGAP, by $\mathrm{Ca} 2+/$ calmodulin-dependent protein kinase II, The Journal of biological chemistry, 279 (2004) 17980-17988.

[13] D.T. Pak, S. Yang, S. Rudolph-Correia, E. Kim, M. Sheng, Regulation of dendritic spine morphology by SPAR, a PSD-95-associated RapGAP, Neuron, 31 (2001) 289-303.

[14] D.T. Pak, M. Sheng, Targeted protein degradation and synapse remodeling by an inducible protein kinase, Science (New York, N.Y.), 302 (2003) 1368-1373.

[15] D.P. Seeburg, M. Feliu-Mojer, J. Gaiottino, D.T. Pak, M. Sheng, Critical role of CDK5 and Polo-like kinase 2 in homeostatic synaptic plasticity during elevated activity, Neuron, 58 (2008) 571-583.

[16] A.C. McMahon, M.W. Barnett, T.S. O'Leary, P.N. Stoney, M.O. Collins, S. Papadia, J.S. Choudhary, N.H. Komiyama, S.G. Grant, G.E. Hardingham, D.J. Wyllie, P.C. Kind, SynGAP isoforms exert opposing effects on synaptic strength, Nature communications, 3 (2012) 900.

[17] K.J. Lee, Y. Lee, A. Rozeboom, J.Y. Lee, N. Udagawa, H.S. Hoe, D.T. Pak, Requirement for Plk2 in orchestrated ras and rap signaling, homeostatic structural plasticity, and memory, Neuron, 69 (2011) 957-973. 
[18] E.F. Johnson, K.D. Stewart, K.W. Woods, V.L. Giranda, Y. Luo, Pharmacological and functional comparison of the polo-like kinase family: insight into inhibitor and substrate specificity, Biochemistry, 46 (2007) 9551-9563.

[19] P.V. Hornbeck, J.M. Kornhauser, S. Tkachev, B. Zhang, E. Skrzypek, B. Murray, V. Latham, M. Sullivan, PhosphoSitePlus: a comprehensive resource for investigating the structure and function of experimentally determined post-translational modifications in man and mouse, Nucleic acids research, 40 (2012) D261-270.

[20] M. Salvi, E. Trashi, G. Cozza, C. Franchin, G. Arrigoni, L.A. Pinna, Investigation on PLK2 and PLK3 substrate recognition, Biochimica et biophysica acta, 1824 (2012) 1366-1373.

[21] M. Salvi, E. Trashi, O. Marin, A. Negro, S. Sarno, L.A. Pinna, Superiority of PLK-2 as alpha-synuclein phosphorylating agent relies on unique specificity determinants, Biochemical and biophysical research communications, 418 (2012) 156-160.

[22] C. Franchin, L. Cesaro, L.A. Pinna, G. Arrigoni, M. Salvi, Identification of the PLK2dependent phosphopeptidome by quantitative proteomics [corrected], PloS one, 9 (2014) e111018.

[23] P.V. Hornbeck, B. Zhang, B. Murray, J.M. Kornhauser, V. Latham, E. Skrzypek, PhosphoSitePlus, 2014: mutations, PTMs and recalibrations, Nucleic acids research, 43 (2015) D512-520.

[24] G. Kauselmann, M. Weiler, P. Wulff, S. Jessberger, U. Konietzko, J. Scafidi, U. Staubli, J. Bereiter-Hahn, K. Strebhardt, D. Kuhl, The polo-like protein kinases Fnk and Snk associate with a $\mathrm{Ca}(2+)$ - and integrin-binding protein and are regulated dynamically with synaptic plasticity, The EMBO journal, 18 (1999) 5528-5539.

[25] R. Dhavan, L.H. Tsai, A decade of CDK5, Nature reviews. Molecular cell biology, 2 (2001) 749-759.

[26] S.G. McCormack, R.L. Stornetta, J.J. Zhu, Synaptic AMPA receptor exchange maintains bidirectional plasticity, Neuron, 50 (2006) 75-88.

[27] Y. Zhu, D. Pak, Y. Qin, S.G. McCormack, M.J. Kim, J.P. Baumgart, V. Velamoor, Y.P. Auberson, P. Osten, L. van Aelst, M. Sheng, J.J. Zhu, Rap2-JNK removes synaptic AMPA receptors during depotentiation, Neuron, 46 (2005) 905-916.

[28] A. Kielland, G. Bochorishvili, J. Corson, L. Zhang, D.L. Rosin, P. Heggelund, J.J. Zhu, Activity patterns govern synapse-specific AMPA receptor trafficking between deliverable and synaptic pools, Neuron, 62 (2009) 84-101.

[29] P.J. Stork, Does Rap1 deserve a bad Rap?, Trends in biochemical sciences, 28 (2003) 267275. 


\section{Tables}

Table 1 Identification of Plk2 Phosphorylation Sites in R-synGAP

\begin{tabular}{|c|c|c|c|c|c|c|c|c|}
\hline \multirow{3}{*}{$\begin{array}{l}\text { Residue } \\
\text { modified }\end{array}$} & \multicolumn{4}{|c|}{$-\mathrm{Ca}^{2+} / \mathrm{CaM}$} & \multicolumn{4}{|c|}{$+\mathrm{Ca}_{2+} / \mathrm{CaM}$} \\
\hline & \multicolumn{2}{|c|}{2 Minutes } & \multicolumn{2}{|c|}{10 Minutes } & \multicolumn{2}{|c|}{2 Minutes } & \multicolumn{2}{|c|}{10 Minutes } \\
\hline & MS2 & MS3 & MS2 & MS3 & MS2 & MS3 & MS2 & MS3 \\
\hline Ser-140 & $\mathrm{X}^{*}$ & & $\mathrm{X}^{*}$ & & $\mathrm{X}^{*}$ & & $\mathrm{X}^{*}$ & \\
\hline Ser-737 & & & & & & & $\mathrm{X}^{* *}$ & \\
\hline Ser-750 & $\mathrm{X}^{*}$ & $\mathrm{X}^{*}$ & $\mathrm{X}^{*}$ & $\mathrm{X}^{*}$ & $\mathrm{X}^{* *}$ & $\mathrm{X}^{*}$ & $\mathrm{X}^{*}$ & $\mathrm{X}^{* *}$ \\
\hline Ser-751 & $\mathrm{X}^{* *}$ & & $\mathrm{X}^{* *}$ & $\mathrm{X}^{* *}$ & $\mathrm{X}^{* *}$ & & $\mathrm{X}^{* *}$ & \\
\hline Ser-756 & $\mathrm{X}^{* *}$ & $X^{*}$ & & & $\mathrm{X}^{* *}$ & & & \\
\hline Ser-765 & $\mathrm{X}^{* *}$ & & $\mathrm{X}^{* *}$ & & $\mathrm{X}^{* *}$ & & $\mathrm{X}^{*}$ & \\
\hline Ser-808 & $X$ & & $X^{*}$ & & $X$ & & $\mathrm{X}^{*}$ & \\
\hline Ser-821 & & & $\mathrm{X}^{* *}$ & & & & $\mathrm{X}$ & \\
\hline Ser-825 & & & $\mathrm{X}^{* *}$ & $X$ & & & $\mathrm{X}^{* *}$ & $\mathrm{X}^{* *}$ \\
\hline Ser-827 & & & $\mathrm{X}^{*}$ & & & & $X$ & \\
\hline Thr-897 & & & $X^{* *}$ & $\mathrm{X}^{*}$ & $\mathrm{X}^{* *}$ & $\mathrm{X}$ & $\mathrm{X}^{* *}$ & $\mathrm{X}^{* *}$ \\
\hline Ser-1099 & & & & & & & $\mathrm{X}^{* *}$ & \\
\hline Ser-1123 & & & & & $X$ & & $\mathrm{X}^{* *}$ & \\
\hline Ser-1283 & & & & & & & $\mathrm{X}^{* *}$ & $\mathrm{X}^{* *}$ \\
\hline
\end{tabular}

Table 2 Identification of CDK5 Phosphorylation Sites in R-synGAP

\begin{tabular}{|c|c|c|c|c|c|c|c|c|}
\hline \multirow{3}{*}{$\begin{array}{l}\text { Residue } \\
\text { modified }\end{array}$} & \multicolumn{4}{|c|}{$-\mathrm{Ca}^{2+} / \mathrm{CaM}$} & \multicolumn{4}{|c|}{$+\mathrm{Ca}_{2+} / \mathrm{CaM}$} \\
\hline & \multicolumn{2}{|c|}{2 Minutes } & \multicolumn{2}{|c|}{10 Minutes } & \multicolumn{2}{|c|}{2 Minutes } & \multicolumn{2}{|c|}{10 Minutes } \\
\hline & MS2 & MS3 & MS2 & MS3 & MS2 & MS3 & MS2 & MS3 \\
\hline Ser-728 & $\mathrm{X}^{* *}$ & $\mathrm{X}^{* *}$ & $\mathrm{X}^{* *}$ & $\mathrm{X}^{* *}$ & $\mathrm{X}^{* *}$ & & $\mathrm{X}^{* *}$ & $\mathrm{X}^{* *}$ \\
\hline Ser-751 & & & & & & & $\mathrm{X}^{* *}$ & \\
\hline Ser-765 & & & & & & & $\mathrm{X}^{* *}$ & \\
\hline Ser-773 & $\mathrm{X}^{* *}$ & $\mathrm{X}^{* *}$ & $\mathrm{X}^{* *}$ & $\mathrm{X}^{* *}$ & $\mathrm{X}^{* *}$ & $\mathrm{X}^{* *}$ & $\mathrm{X}^{*}$ & $\mathrm{X}^{* *}$ \\
\hline Thr-775 & $X$ & $\mathrm{X}$ & $\mathrm{X}$ & $\mathrm{X}$ & & & $\mathrm{X}^{*}$ & $\mathrm{X}$ \\
\hline Ser-802 & $X$ & $\mathrm{X}$ & $\mathrm{X}$ & $X$ & $X$ & $X$ & $X$ & $X$ \\
\hline Ser-842 & $\mathrm{X}^{* *}$ & & $\mathrm{X}^{*}$ & $X^{*}$ & $\mathrm{X}^{* *}$ & & $\mathrm{X}^{* *}$ & $\mathrm{X}^{*}$ \\
\hline Thr-897 & & & & & $\mathrm{X}^{*}$ & $\mathrm{X}^{* *}$ & $\mathrm{X}^{* *}$ & $\mathrm{X}^{* *}$ \\
\hline Ser-1093 & & & & & & & $\mathrm{X}^{*}$ & \\
\hline Ser-1099 & & & & & & & $\mathrm{X}^{* *}$ & $\mathrm{X}^{* *}$ \\
\hline Ser-1123 & & & & & & & $\mathrm{X}^{* *}$ & \\
\hline
\end{tabular}




\section{Table Legend}

X marks Plk2 (Table 1) or CDK5 (Table 2) phosphorylation sites in r-synGAP identified as described under Methods. Phosphorylated residues are categorized according to the length of the phosphorylation reaction ( 2 minutes, 10 minutes), presence/absence of $\mathrm{Ca}^{2+} / \mathrm{CaM}$ in the phosphorylation reaction and their detection in MS2 or MS3 spectra. The sites highlighted with one or two asterisks have phosphorylation false localization rates less than 5\% or 1\%, respectively (predicted as described in [4]).

\section{Figures}

Fig. 1.
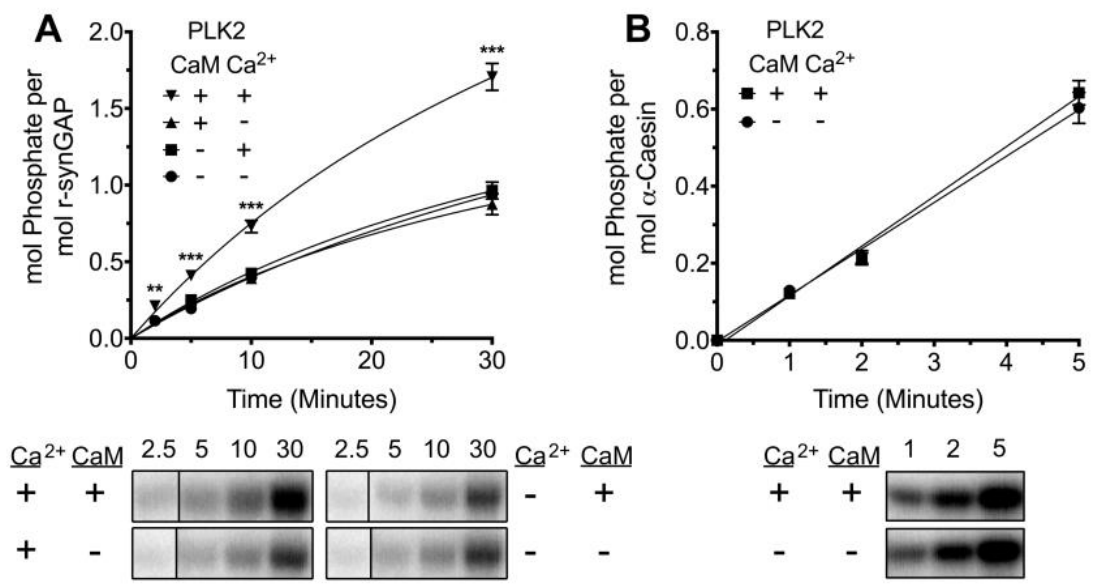

Fig. 2.
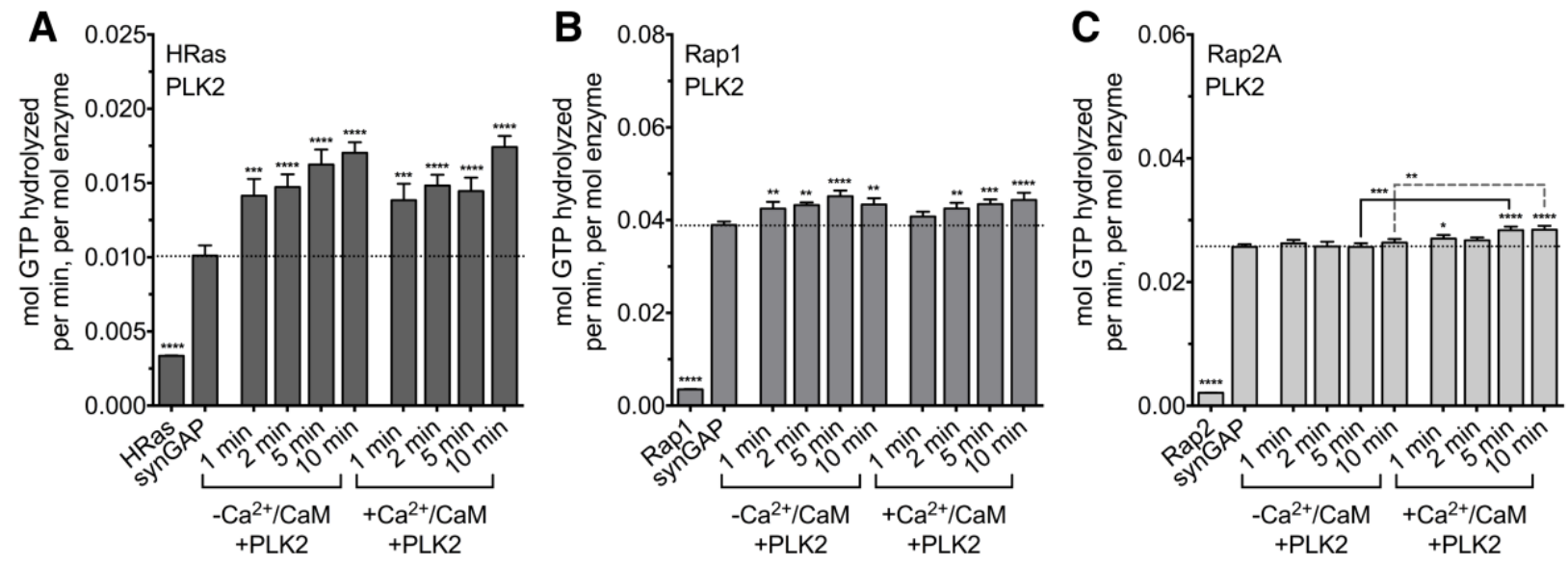
Fig. 3.

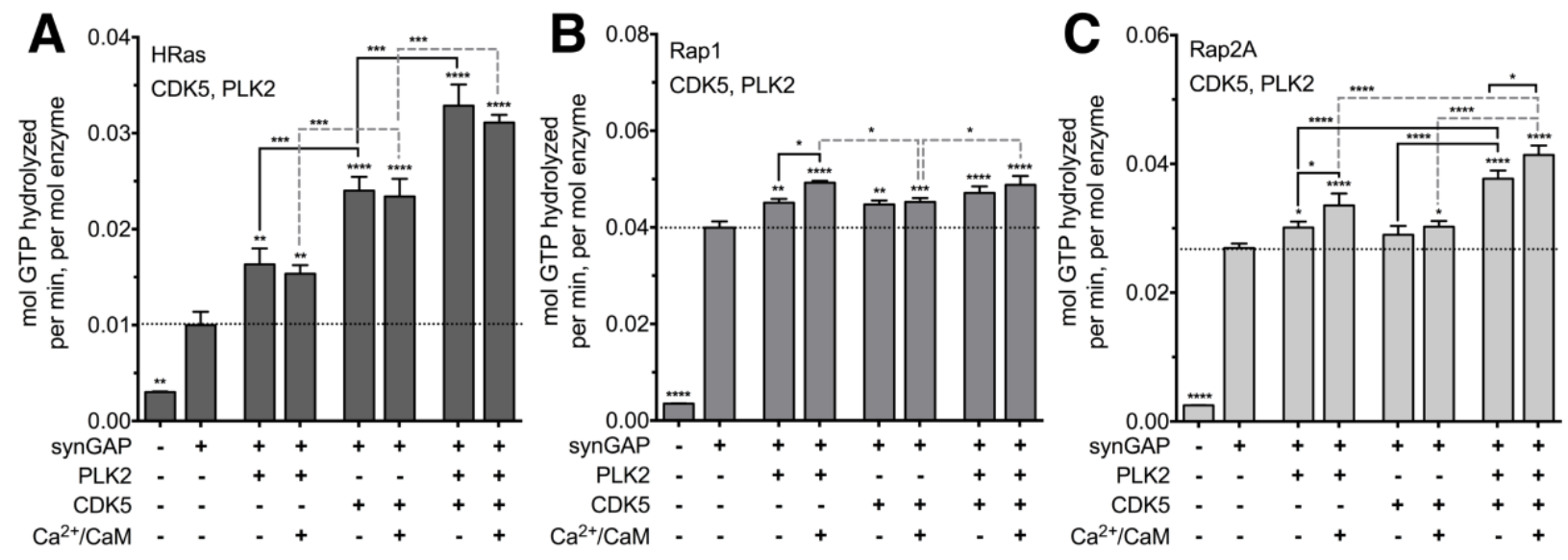

Fig. 4.
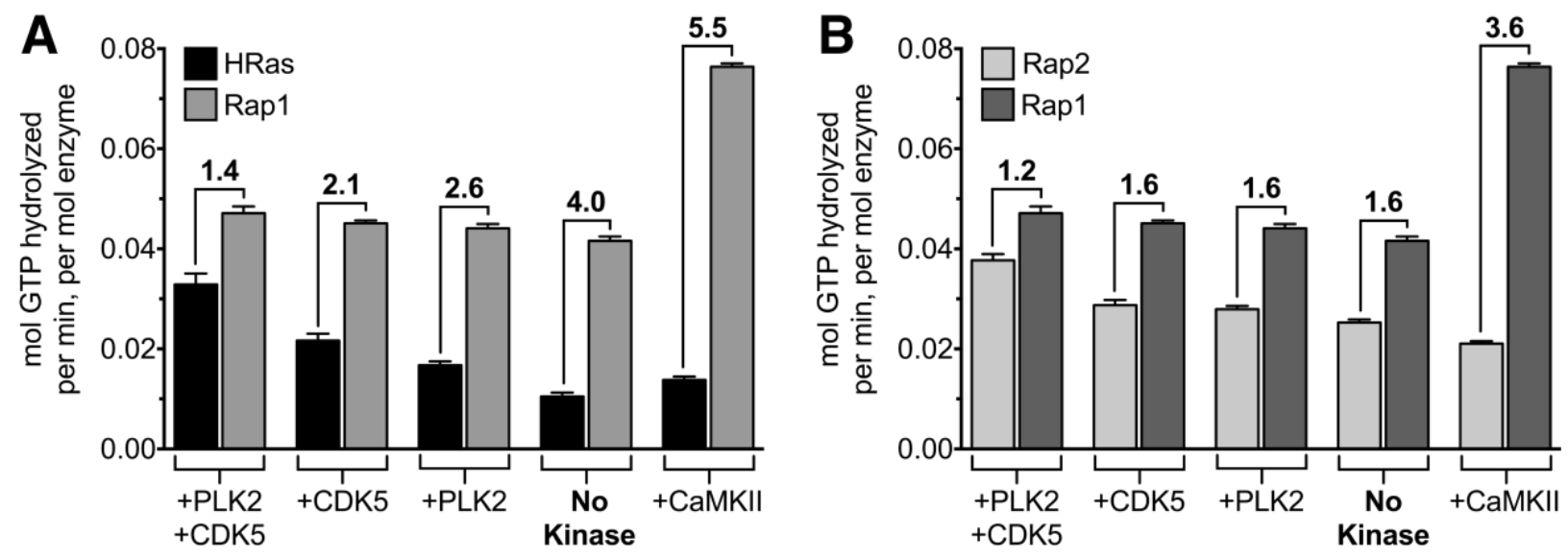

Figure Legends

Fig. 1. Effect of $\mathrm{Ca}^{2+} / \mathrm{CaM}$ on Stoichiometry of Phosphorylation of R-synGAP by Plk2. Stoichiometry of phosphorylation of (A) r-synGAP and (B) dephosphorylated $\alpha$-casein by Plk2. R-synGAP (286 nM) or dephosphorylated $\alpha$-casein $(3.7 \mu \mathrm{M})$ were incubated in the presence of $110 \mathrm{nM}$ Plk2, $500 \mu \mathrm{M}\left[\gamma-{ }^{32} \mathrm{P}\right]$-ATP (375 cpm/pmol), 0 or $0.7 \mathrm{mM} \mathrm{CaCl} 2$ and 0 or $3.4 \mu \mathrm{M} \mathrm{CaM}$, as described in [4]. All data are mean \pm s.e.m. $(n=4-7)$. The statistical significance of differences from the phosphorylation of r-synGAP in the presence of $\mathrm{Ca}^{2+}$ and $\mathrm{CaM}$ was determined by ordinary one way ANOVA (uncorrected Fisher's LSD). **, $\mathrm{p}<0.01 ; * * *, \mathrm{p}<0.001$. 
Fig. 2. Effect of Plk2 Phosphorylation on GAP Activity of R-synGAP. R-synGAP (725 nM) was phosphorylated for 1 to $10 \mathrm{~min}$ in the presence of $30 \mu \mathrm{M} \mathrm{ATP}, 0$ or $0.7 \mathrm{mM} \mathrm{CaCl}, 0$ or $3.4 \mu \mathrm{M} \mathrm{CaM}$ and $230 \mathrm{nM}$ Plk2 as described in [4]. GAP activities of control and phosphorylated r-synGAP (250 nM) were then measured by incubation with [ $\left.\mathrm{\gamma}^{-}{ }^{32} \mathrm{P}\right]-\mathrm{GTP}-$ loaded GTPases for $10 \mathrm{~min}$ at $25^{\circ} \mathrm{C}$ as described in [4]. (A) HRas GAP activity; (B) Rap1 GAP activity; and (C) Rap2 GAP activity following phosphorylation by Plk2. Initial rates were measured at $10 \mathrm{~min}$; GTPase activity was linear for 25 min (data not shown). Data are mean \pm s.e.m $(n=7-10)$. The statistical significance of differences from the GTPase activity of unphosphorylated r-synGAP (No Kinase) was determined by ordinary one way ANOVA (uncorrected Fisher's LSD). *, $\mathrm{p}<0.05 ; * *, \mathrm{p}<0.01 ; * * *, \mathrm{p}<0.001 ; * * * *, \mathrm{p}<0.0001$. Dotted lines indicate the levels of GTPase activity in the presence of unphosphorylated synGAP.

Fig. 3. Effect of Concurrent CDK5 and Plk2 Phosphorylation on GAP Activity of R-synGAP. R-synGAP was phosphorylated as described in Fig. 2 and [4] using 230 nM CDK5/p35, 230 nM Plk2 or 230 nM CDK5/p35 and Plk2. (A) HRas GAP activity; (B) Rap1 GAP activity; and (C) Rap2 GAP activity following phosphorylation by CDK5, Plk2 or both enzymes. Data are mean \pm s.e.m $(n=10)$. The statistical significance of differences from the GTPase activity of unphosphorylated r-synGAP (No Kinase) was determined by ordinary one way ANOVA (uncorrected Fisher's LSD). *, p<0.05; **, p<0.01; ***, $\mathrm{p}<0.001 ; * * * *, \mathrm{p}<0.0001$. Dotted lines indicate the levels of GTPase activity in the presence of unphosphorylated synGAP.

Fig. 4. Alteration of the ratios of (A) HRas to Rap1 and (B) Rap2 to Rap1 GAP activities of r-synGAP after phosphorylation by Plk2, CDK5, Plk2 and CDK5 or CaMKII. Data from [4] and Fig. 2 and Fig. 3 were combined and re-plotted to emphasize the change in ratios of HRas to Rap1 or Rap2 to Rap1 GAP activities after phosphorylation of r-synGAP for 10 min by Plk2, CDK5, Plk2 and CDK5 or CaMKII. The numbers above each bracket are the ratios of the means of (A) Rap1 to HRas or (B) Rap2 to Rap1 GAP activity for each pair of conditions. 


\section{Appendix A. Supplementary Data}

Supplementary data related to this article is attached as a separate document. 